\title{
Correction to: Huddling remodels gut microbiota to reduce energy requirements in a small mammal species during cold exposure
}

Xue-Ying Zhang ${ }^{1,2+}$, Gansukh Sukhchuluun ${ }^{1,2+}$, Ting-Bei Bo ${ }^{1,2 \dagger}$, Qing-Sheng Chi ${ }^{1}$, Jun-Jie Yang ${ }^{3}$, Bin Chen ${ }^{3}$, Lei Zhang ${ }^{3,4^{*}}$ and De-Hua Wang ${ }^{1,2^{*}}$

\section{Correction}

Following publication of the original article [1], the authors reported an error in the caption of Fig. 4.

Originally, the incorrect figure caption was published as:

Fig. 4 Effects of huddling and cold on the concentrations of short-chain fatty acids (SCFAs). The concentrations of acetic acid (a), propionic acid (b), butyric acid (c), isobutyric acid (d), valeric acid (e), and isovaleric acid (f) in huddling and separated Brandt's voles at warm and cold $T_{\mathrm{a}}(n=7-9 /$ group). ${ }^{*} P<0.05$

The correct caption is:

Fig. 4 Effects of huddling and cold on the concentrations of short-chain fatty acids (SCFAs). The concentrations of acetic acid, propionic acid, butyric acid, isobutyric acid, valeric acid, and isovaleric acid in huddling and separated Brandt's voles at warm and cold $T_{\mathrm{a}}(n=7-9 /$ group). ${ }^{*} P<0.05$

\footnotetext{
Author details

'State Key Laboratory of Integrated Management of Pest Insects and Rodents, Institute of Zoology, Chinese Academy of Sciences, Beijing 100101, China. ${ }^{2}$ University of Chinese Academy of Sciences, Beijing 100049, China. ${ }^{3}$ College of Life Science, Shandong Normal University, Ji'nan 250014, China. ${ }^{4}$ Microbiome Research Center, Shandong Institutes for Food and Drug Control, Ji'nan 250101, China.
}

Published online: 09 July 2018

\section{Reference}

1. Zhang, et al. Huddling remodels gut microbiota to reduce energy requirements in a small mammal species during cold exposure.

Microbiome. 2018;6:103. https://doi.org/10.1186/s40168-018-0473-9.

\footnotetext{
* Correspondence: wangdh@ioz.ac.cn

'Xue-Ying Zhang, Gansukh Sukhchuluun and Ting-Bei Bo contributed equally to this work.
} 